\title{
Optimal Performance for Solar Thermal Power System
}

\author{
Jianfeng LU $^{1}$, Jing DING ${ }^{1}$, Jianping YANG ${ }^{2}$ \\ ${ }^{1}$ School of Engineering, Sun Yat-Sen University, Guangzhou, China \\ ${ }^{2}$ Key Laboratory of Enhanced Heat Transfer and Energy Conservation of the Ministry of Education, South \\ China University of Technology, Guangzhou China \\ Email:liujf01@mails.tsinghua.edu.cn
}

\begin{abstract}
Solar thermal power is currently one of the important trends and research hotspots of solar energy. In present paper, basic physical model is proposed to investigate the solar thermal power, and the operating temperature is optimized to maximize the electricity generating efficiency. When the concentrated energy flux rises, the absorption efficiency of heat receiver will first increase and then decrease, while the increasing of flow velocity can improve the absorption performance. As the working temperature rising, the heat loss of infrared radiation and natural convection increases quickly, so the absorption efficiency obviously decreases, while the Carnot efficiency of the steam turbine cycle will rise. Because of the coupling effects of the heat absorption cycle and turbine cycle, the electricity generating efficiency will reach maximum with the optimal working temperature.
\end{abstract}

Keywords: solar energy, heat receiver, operating temperature, electricity generating efficiency

\section{1. 引言}

太阳能作为最丰富和可广泛获取的可再生能源形 式, 对解决能源危机和环境问题有特别重要的作用。 太阳能热发电系统主要由聚光系统、吸热传热系统和 发电系统构成 ${ }^{[1]}$ 。太阳能热发电系统的发电系统类似 常规电站, 其效率受限于 Carnot 循环效率, 主要由介 质工作温度决定。

吸热器是太阳能光热转化的核心部件, 其工作温 度和吸热效率直接影响电站的发电效率, 因此得到广 泛的关注和研究。Fujiwara 等 $^{[2]}$ 实验研究了空间太阳 能热利用系统中热管吸热器的热力学性质。吸热器的 能量损失决定系统吸热效率, 主要包括自然对流和辐 射传热。Clausing ${ }^{[3]}$ 分析了腔式吸热器中对流传热引起 的能量损失。Dehghan 与 Behnia ${ }^{[4]}$ 研究了腔式吸热器 中自然对流与辐射的联合作用。通过分析整个热发电 系统, 研究者对吸热器的构型和操作温度进行了优化。 刘志刚等 ${ }^{[5]}$ 设计一种高效率的新型腔式吸热器, 并进 行了实验研究。Steinfeld 与 Schubnell ${ }^{[6]}$ 研究了腔式吸 热器吸热过程的优化尺寸和工作温度。Segal 与 Epstein $^{[7]}$ 根据经验关联式提出了热发电系统的优化操 作温度。目前, 太阳能热发电系统中吸热传热循环与
发电循环的耦合及优化性能还有待深入研究。

本文建立太阳能热发电系统的基本物理模型，由 此分析系统发电效率并优化工作温度。利用太阳能吸 热系统的吸热传热模型，研究吸热管壁面温度与吸热 效率的变化特征, 特别分析聚光热流密度、流速与工 作温度的影响。通过吸热传热循环与发电循环的耦合, 分析发电效率最大时的优化温度。

\section{2. 太阳能热发电系统的物理模型}

太阳能热发电系统的基本热力学循环如图 1 所 示, 主要包括吸热传热循环和发电循环, 聚光系统产 生的高能流辐射可作为吸热器的热边界条件。吸热传 热循环中，工作介质首先被泉从低温蓄热器抽入吸热 器 (进口温度 $T_{f 0}$ ), 然后吸收聚光系统汇聚的太阳光 能提高温度并存入高温蓄热器 (工作温度 $T_{f l}$ ), 高温 介质与发电循环进行热交换降低温度后流回低温蓄热 器。发电循环中, 工作介质通过与吸热传热循环进行 热交换提高温度，然后利用发电机对外做功并发电。

工作介质在吸热传热循环中起重要作用, 常用蓄 热传热介质有水/水蒸气, 空气与熔融盐等。空气介质 具有比较广的工作温度范围, 在高温热发电和小型系统 


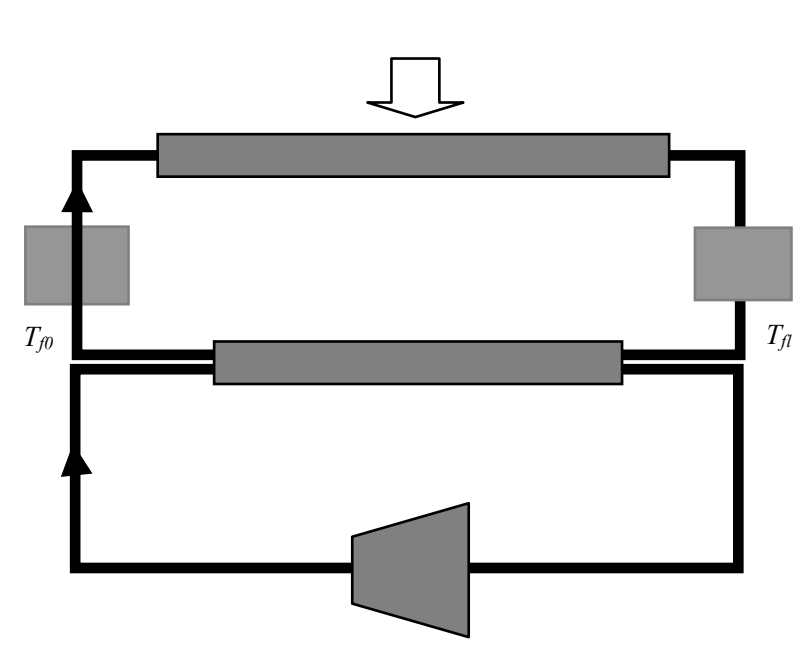

图 1. 太阳能热发电系统

Figure 1. Solar thermal power system

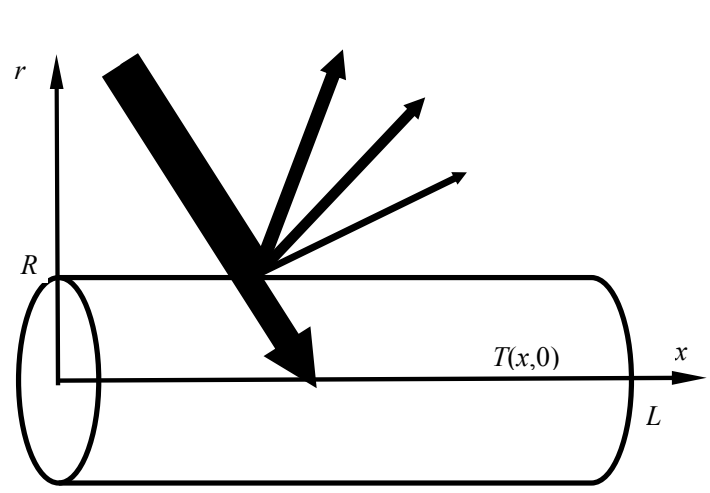

图 2. 吸热管内外的传热过程

Figure 2. The heat transfer process in the heat receiver

中有广泛的应用前景, 可以作为研究热发电的理想模 型。为降低辐射能量损失, 吸热器表面通常涂有太阳 能选择性吸收涂层, 研究者开发了大量的太阳光谱选 择性吸收涂层 ${ }^{[8-9]}$ 。

相比吸热与发电过程的能量损失, 蓄热和传热过 程的能量损失相对较小, 本文主要分析吸热和发电过 程的能量利用效率。为理论简化和分析太阳能热发电 系统中的吸热过程, 研究轴对称直管道的吸热过程。 如图 2 所示, 吸热管半径为 $R$, 表面具有太阳能选择 性吸收涂层, 管壁厚度与热阻忽略。能流密度为 $I$ 的入 射光首先被涂层表面反射，涂层反射系数为 $\mathrm{K}=1-\mathrm{a}$ ， $\alpha$ 为吸收系数。管外传热引起的能量损失主要包括自然 对流与红外辐射, 其中自然对流系数为 $h_{n}$, 红外辐射 发射系数为 $\epsilon$ 。管内吸收的热能通过强迫对流传递。
由上所述，吸热管壁面吸热传热的能量守恒方程 为:

$$
I-\kappa \cdot I=\alpha I=h_{n}\left(T_{w}-T_{s}\right)+\varepsilon \cdot \sigma T_{w}{ }^{4}+q_{f}
$$

其中 $T_{s}$ 为环境温度, $T_{w}$ 为壁面温度, $q_{f}$ 为管内对 流传热热流密度。

对于管内充分发展流，对流换热可表示为：

$$
q_{f}=h_{f} \cdot\left(T_{w}-T_{f}\right)
$$

其中 $h_{f}$ 为强迫对流换热系数, $T_{f}$ 为管内流体温 度。吸热管内流动通常为湍流, 其换热系数可以由实 验关联为 ${ }^{[10]}$ :

$$
\begin{gathered}
h_{f}=\mathrm{Nu} \cdot \frac{k}{D} \\
\mathrm{Nu}=0.0243 \operatorname{Re}^{0.8} \operatorname{Pr}^{0.4}
\end{gathered}
$$


式(2)代入式(1)得

$$
\alpha I=h_{n}\left(T_{w}-T_{s}\right)+\varepsilon \cdot \sigma T_{w}{ }^{4}+h_{f} \cdot\left(T_{w}-T_{f}\right)
$$

管内沿流向的能量方程为

$$
\begin{aligned}
q_{f} \cdot 2 \pi R & =\frac{\partial}{\partial x} \int_{0}^{R} \rho c_{p} T u(r) 2 \pi r d r \\
& =\rho c_{p} \frac{\partial T_{f}}{\partial x} \int_{0}^{R} u(r) 2 \pi r d r=\rho c_{p} \frac{\partial T_{f}}{\partial x} \cdot \pi R^{2} u_{\mathrm{av}}
\end{aligned}
$$

其中 $u_{\mathrm{av}}$ 为管内平均流速。

壁面局部吸热效率和吸热管平均吸热效率分别 为:

$$
\begin{gathered}
\eta_{a b}=\frac{q_{f}}{I}=\frac{h_{f}\left(T_{w}-T_{f}\right)}{I} \\
\overline{\eta_{a b}}=L^{-1} \int_{0}^{L} \eta_{a b} d x
\end{gathered}
$$

发电循环中, 热力学效率主要由介质工作温度 $T_{f l}$ 决定, 初步研究中可简化为 Carnot 循环效率 ${ }^{[11]}$, 即

$$
\eta_{c}=\frac{T_{f l}-T_{s}}{T_{f l}}
$$

太阳能热发电系统中, 聚光效率主要由聚光系统 和辐射条件决定, 与系统工作温度无关。对于已经由 聚光系统会聚到吸热器的太阳光能, 其聚光发电效率 可表示为吸热效率与热力循环效率之积, 即

$$
\eta=\overline{\eta_{a b}} \eta_{c}
$$

以一小型电站为例具体研究太阳能热发电系统的 基本性能和优化参数。吸热管管径 $R=0.01 \mathrm{~m}$, 管长 $L=1 \mathrm{~m}$, 自然对流传热系数和环境温度分别为 $5 \mathrm{Wm}^{-2} \mathrm{~K}^{-1}$ 与 $293 \mathrm{~K}$ 。文中计算采用空气的基本热力学
性质为 $, \rho=1.77 \mathrm{kgm}^{-3}, c_{p}=1006 \mathrm{Jkg}^{-1} \mathrm{~K}^{-1}, \mathrm{Pr}=0.743$, $k=0.0181 \mathrm{Wm}^{-1} \mathrm{~K}^{-1}, \mu=0.0000134 \mathrm{kgm}^{-1} \mathrm{~s}^{-1}$ 。管壁采用 Co-Cd-BT 涂层 ${ }^{[12]}$, 其辐射性能吸收系数 $a=0.96$, 发 射系数 $\epsilon=0.12$ 。

\section{3. 太阳能热发电系统的性能优化}

首先研究吸热管的基本吸热性能, 图 3 表示不同 聚光热流密度下的壁面温度和吸热效率, 其中 $T_{f}=473 \mathrm{~K}, u_{\mathrm{av}}=5.0 \mathrm{~m} / \mathrm{s}$ 。壁面温度随聚光热流密度提高 而线性增加，热流密度为 $20 \mathrm{kWm}^{-2}$ 时壁面温度达到 $900 \mathrm{~K}$ 。低聚光热流密度时, 随聚光热流密度上升, 能 量损失缓慢上升，而吸热效率显著提高。高聚光热流 密度时, 随聚光热流密度上升, 能量损失特别是红外 辐射能量显著提高, 因此吸热效率随之下降。聚光热 流密度在最优值 $12 \mathrm{kWm}^{-2}$ 时，吸热效率达到最大值 $60.8 \%$ 。

图 4 表示不同流速条件下的壁面温度和吸热效 率, 其中 $T_{f}=473 \mathrm{~K}, I=10 \mathrm{kWm}^{-2}$ 。随着管内流速的提高, 管内换热条件明显改善, 壁面温度随着降低, 而吸热 效率显著提高。流速低于 $5 \mathrm{~m} / \mathrm{s}$ 时吸热效率低于 $60 \%$, 严重限制该热发电系统的发电效率。流速高于 $15 \mathrm{~m} / \mathrm{s}$ 时，管内流动换热的热阻较小，吸热效率缓慢增加，但 流动的驱动功率将显著提高。根据吸热器的基本性能, 热发电系统需要优化聚光热流密度和管内对流流速。

太阳能热发电系统中, 吸热传热循环和发电循环 的耦合对聚光能量的发电效率起着关键作用, 其中最 重要的因素为工作温度。工作温度上升时, 吸热器表 面自然对流和红外辐射的热损失明显增加，由此降低吸 热效率, 而发电循环效率则随温度升高而提高, 因此

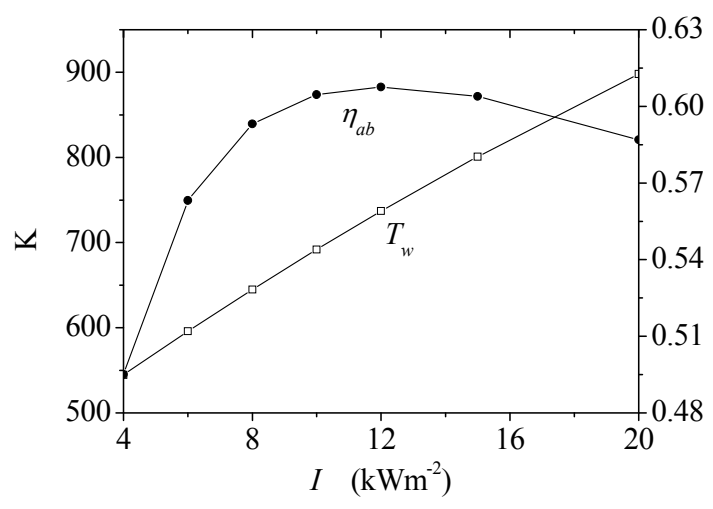

图 3. 不同能流密度下壁面温度和吸热系数分布

Figure 3. The temperature distributions and absorption efficiency under different energy fluxes 


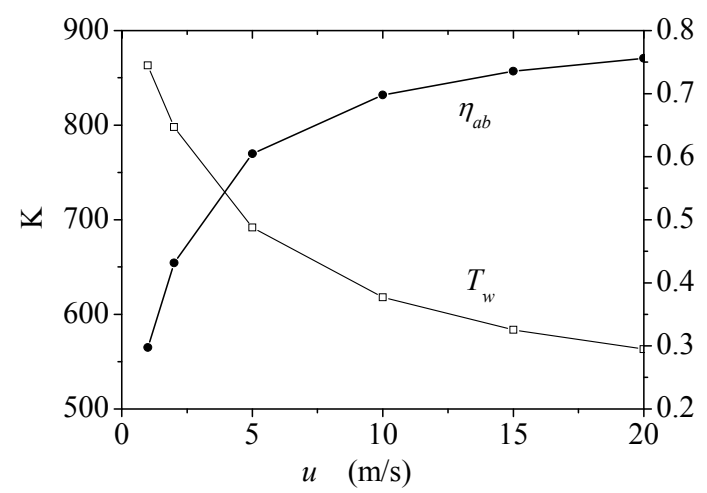

图 4. 不同流速条件下壁面温度分布和吸热系数分布

Figure 4. The temperature distributions and absorption efficiency under different velocities

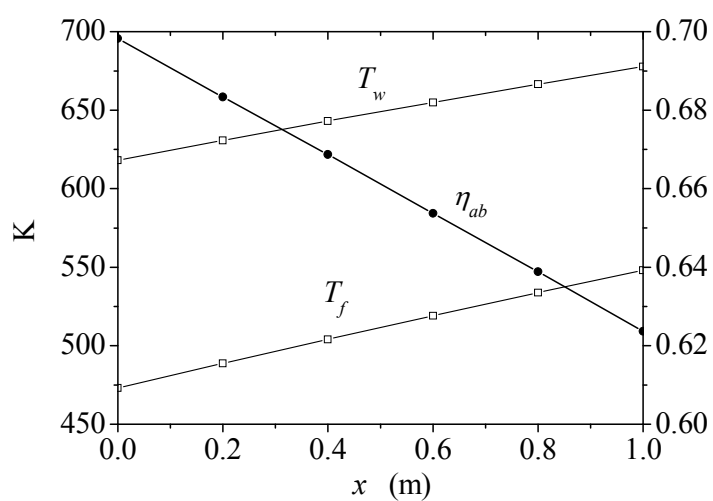

(a) $T_{0}=473 \mathrm{~K}$

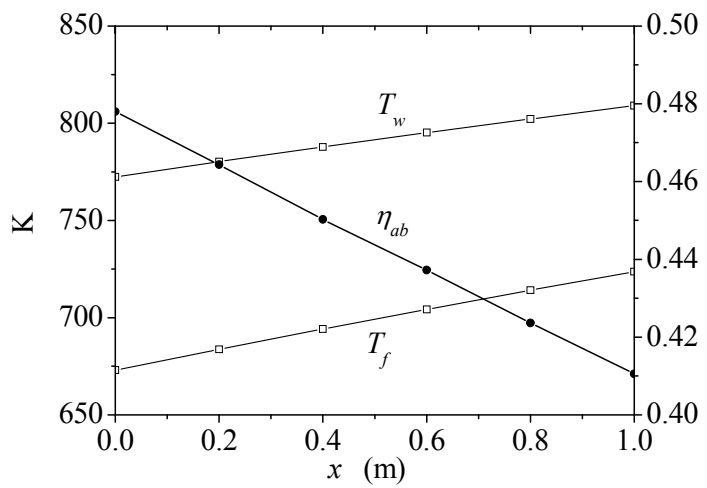

(b) $T_{10}=673 \mathrm{~K}$

图 5. 不同进口温度下的壁面温度和吸热系数分布

Figure 5. The temperature distributions and absorption efficiency under different inlet temperatures

存在系统的优化状态。考虑吸热器进口温度分别为 $T_{f 0}=473 \mathrm{~K}$ 或 $673 \mathrm{~K}$, 则吸热管沿程壁面温度和吸热效 率分布如图 5 , 其中 $u_{\mathrm{av}}=10.0 \mathrm{~m} / \mathrm{s}, I=10 \mathrm{kWm}^{-2}$ 。沿着流 线方向, 壁面温度和主流温度逐渐增加, 而吸热效率 则逐渐降低。吸热器进口温度与工作温度上升时, 吸 热器的平均吸热效率显著降低。吸热器进口温度由
$473 \mathrm{~K}$ 提高到 $673 \mathrm{~K}$ 时 吸热器的平均吸热效率由 $66.1 \%$ 降低至 44.4\% ,同时其工作温度从 $678 \mathrm{~K}$ 提高到 $809 \mathrm{~K}$ 。 由式 (6) 和 (7)，吸热管吸热效率和发电循环效 率随工作温度的变化情况图 6 , 其中 $u_{\mathrm{av}}=10.0 \mathrm{~m} / \mathrm{s}$, $I=10 \mathrm{kWm}^{-2}$ 。随着工作温度提高, 自然对流和红外辐 射引起的热损失增加，吸热管吸热效率显著降低，工 


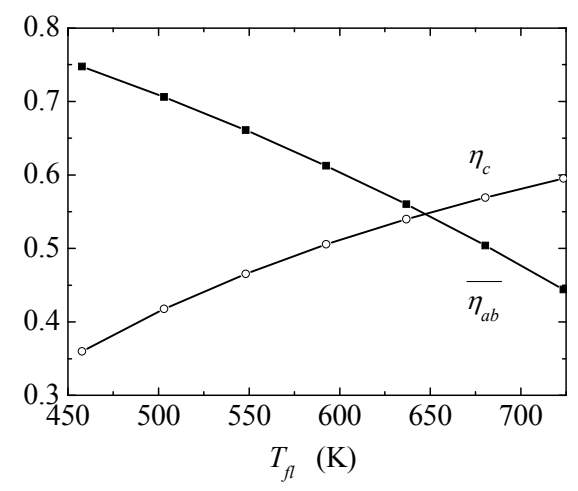

图 6. 不同工作温度下吸热效率和 Carnot 效率

Figure 6. The absorption and Carnot efficiencies under different working temperatures

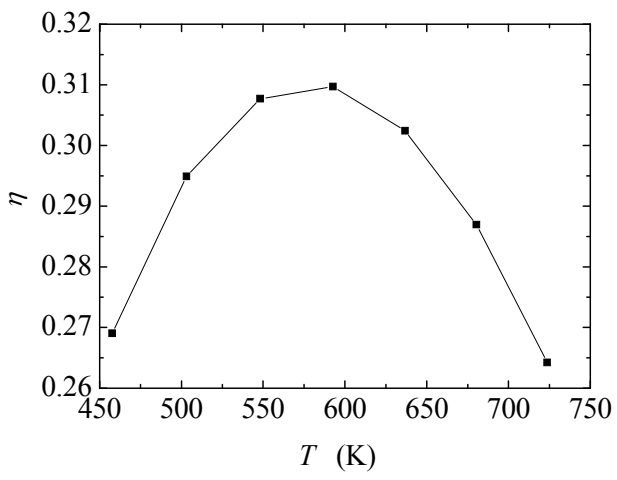

图 7. 不同工作温度下聚光发电效率

Figure 7. The electricity generating efficiency under different working temperatures

作温度由 $458 \mathrm{~K}$ 提高到 $724 \mathrm{~K}$ 时吸热器平均吸热效率由 $74.8 \%$ 降低至 $44.4 \%$ 。另一方面, 发电循环效率则随工 作温度的提高而显著上升, 工作温度由 $458 \mathrm{~K}$ 提高到 $724 \mathrm{~K}$ 时发电循环效率由 $36.0 \%$ 升至 59.5\%。

图 7 为太阳能热发电系统聚光发电效率随工作温 度变化情况, 其中 $u_{\mathrm{av}}=10.0 \mathrm{~m} / \mathrm{s}, I=10 \mathrm{kWm}^{-2}$ 。工作温 度较低时, 发电循环效率的提高起主导作用, 因此聚 光发电效率随工作温度上升而提高。工作温度较高时, 吸热器吸热效率随温度升高而下降起主要作用, 因此 聚光发电效率随工作温度上升而降低。由于发电循环 和吸热传热的耦合, 优化工作温度为 $590 \mathrm{~K}$, 此时聚光 发电效率达最大值 31.0\%。由图 3-4, 聚光能流密度和 流速显著影响吸热效率和壁面温度, 因此可以进一步 影响优化温度和发电效率。

\section{4. 结 论}

本文建立了太阳能热发电系统的基本物理模型， 并通过分析吸热传热循环与发电循环的耦合导出优化
工作温度。文中首先研究具有太阳能选择性吸收涂层 的聚光太阳能吸热管的吸热传热特性。随着入射光能 增加，吸热管壁面温度随之线性升高，而吸热效率则 先增加后减少。增加流速可以提高吸热管性能，吸热 效率随流速增加而减少，而管壁温度则显著下降。随 着工作温度提高, 自然对流和红外辐射引起的热损失 增加，因此吸热管吸热效率显著降低。发电循环效率 的变化趋势与吸热效率相反, 随工作温度升高而迅速 提高。由于发电循环与吸热传热循环的耦合, 聚光发 电效率在工作温度为优化温度时达最大值。

\section{REFERENCES}

[1] S. A. Kalogirou, "Solar thermal collectors and applications," Progress in Energy and Combustion Science, Vol. 30, pp. 231-295, 2004.

[2] M. Fujiwara, T. Sano, K. Suzuki, et al., "Thermal analysis and fundamental tests heat pipe receiver for solar dynamic space system," Journal of Solar Energy Engineering, Vol. 112, pp. 177-182, 1990. 
[3] A. Clausing, "Analysis of convective losses from cavity solar central receivers," Solar Energy, Vol. 27, pp. 295300, 1981.

[4] A. A. Dehghan and M. Behnia, "Combined natural convection conduction and radiation heat transfer in a discretely heated open cavity," ASME Journal of Heat Transfer, Vol. 118, pp. 54-56, 1996.

[5] Z. G. Liu, C. P. Zhang, Y. H. Zhao, and D. W. Tang, "The design and experiments of a new cavity absorber," Acta Energiae Solaris Sinica, Vol. 26, pp. 332-337, 2005. (in Chinese)

[6] A. Steinfeld and M. Schubnell, "Optimum aperture size and operating temperature of a solar cavity-receiver," Solar Energy, Vol. 50, pp. 19-25, 1993.

[7] A. Segal and M. Epstein, "Optimized working temperatures of a solar central receiver," Solar Energy, Vol. 75, pp. 503-510, 2003.

[8] C. E. Kennedy, "Review of mid- to high-temperature solar selective absorber materials," NREL/TP-520-31267, 2002.

[9] Y. Sun, Y. Y. Shi, and F. C. Wang, "Study on thermal stability of solar selective absorbing surfaces with new material," Acta Energiae Solaris Sinica, Vol. 23, pp. 39-42, 2002. (in Chinese)

[10] J. H Lienhard IV and J. H. Lienhard V, "A heat transfer textbook," Phlogiston Press, Chambridge, Massachusetts, U.S.A, 2002

[11] R. T. Stephen, "Thermodynamics: concepts and applications," Cambridge University Press, New York, 2006

[12] L. Cindrella, "The real utility ranges of the solar selective coatings," Solar Energy Materials and Solar Cells, Vol. 23, pp. 1898-1901, 2007. 